\title{
Evaluation of Tests for Separability and Symmetry of Spatio-temporal Covariance Function
}

\author{
Elham Behshad and Mohsen Mohammadzadeh* \\ Tarbiat Modares University
}

\begin{abstract}
In recent years, some investigations have been carried out to examine the assumptions like stationarity, symmetry and separability of spatio-temporal covariance function; which would considerably simplify fitting a valid covariance model to the data by parametric and nonparametric methods. In this article, assuming a Gaussian random field, we consider the likelihood ratio separability test, a variety of nonparametric and spectral tests of symmetry and also separability of spatio-temporal covariance function. Comparing the tests of symmetry and separability in a level of scenarios, the best ones would be introduced.
\end{abstract}

Keywords. Spatio-temporal data; stationarity; symmetry; stationary; separability.

MSC 2010: 62H11.

\section{Introduction}

Analysis of spatial-temporal data is a principle objective in many sciences; an example is geostatistics. In the recent decade there have been literatures on correlation structure and modeling of spatio-temporal data. The covariance function detecting the correlation structure of the data is unknown and should be estimated. One way to alleviate the computational burden due to

* Corresponding author 
large size of spatio-temporal data is to impose certain structure on the covariance function, such as stationary, full symmetry and separability, which greatly reduce the computation time of the covariance matrix. Although symmetry and separability are desirable assumptions from a computational point of view, They may not be appropriate for some real data sets. Examples of this are described by Li et al. (2007) on lack of symmetry and separability for two data set of pacific ocean wind data described by Cressie and Huang (1999) and the Irish wind data described by Haslett and Reftary (1989).

Let $\left\{Z(\mathbf{s}, t): \mathbf{s} \in D \subseteq R^{d}, t \in T \subseteq R\right\}$ be a strictly stationary spatiotemporal random field with covariance function $C(\mathbf{h}, u)$, where $\mathbf{h}$ and $u$ are spatial and time lags. A spatio-temporal covariance function is fully symmetry if

$$
C(\mathbf{h}, u)=C(-\mathbf{h}, u)=C(\mathbf{h},-u)=C(-\mathbf{h},-u), \quad(\mathbf{h}, u) \in D \times T .
$$

Park and Fuentes (2008) express two types of axial symmetry in time and space. A spatio-temporal covariance function is axially symmetric in time if

$$
C(\mathbf{h}, u)=C(\mathbf{h},-u),
$$

and it is $k$-axially symmetric in space if

$$
C(\mathbf{h}, u)=C\left(h_{1}, \ldots, h_{k-1},-h_{k}, h_{k+1}, \ldots, h_{d}, u\right) .
$$

A spatio-temporal covariance function is separable if and only if $C(\mathbf{h}, u)=$ $C_{S}(\mathbf{h}) C_{T}(u)$, where $C_{S}(\cdot)$ and $C_{T}(\cdot)$ are pure spatial and pure temporal covariance functions respectively, or equivalently

$$
\frac{C(\mathbf{h}, u)}{C(\mathbf{h}, 0)}=\frac{C(\mathbf{0}, u)}{C(\mathbf{0}, 0)} .
$$

The separability can be expressed based on the Kronecker product of space and time covariance matrices. If $\mathbf{U}=\left(u_{i j}\right)$ is an $n \times n$ space covariance matrix and $\mathbf{V}=\left(v_{i j}\right)$ is an $m \times m$ temporal covariance matrix, then the covariance function is separable if and only if

$$
\Sigma=\mathbf{U} \otimes \mathbf{V}=\left(\begin{array}{ccc}
u_{11} \mathbf{V} & \ldots & u_{1 n} \mathbf{V} \\
\vdots & & \vdots \\
u_{n 1} \mathbf{V} & \ldots & u_{n n} \mathbf{V}
\end{array}\right)
$$


In literature, many approaches has been proposed for symmetry and separability tests of spatio-temporal covariance functions. Lu and Zimmermann (2005) proposed a likelihood ratio statistic for separability; also Mitchell et al. (2006) proposed a formal test of separability based on a likelihood ratio statistic that was context of multivariate repeated measures. While, Fuentes (2006) developed a nonparametric test of separability based on spectral representation of a spatio-temporal covariance. Li et al. (2007) considered a unified framework for testing various assumptions commonly made for stationary covariance functions based on the asymptotic normality of spatiotemporal covariance estimators. Shao and Li (2009) proposed two estimator for the asymptotic covariance matrix of Li's test statistic. Park and Fuentes (2008) extended the spectral method to detect symmetry of spatio-temporal covariance function. In this paper simulation studies were carried out to compare the sizes and powers of the proposed testing hypothesis for various conditions. Then the most powerful tests of symmetry and separability were chosen. Finally, the chosen tests were applied for wind-speed data. All the computations were done with $\mathrm{R}$ software and the provided $\mathrm{R}$-cods are available via authors.

\section{Tests of Symmetry and Separability}

In this section, the symmetry and separability tests of covariance functions which were proposed in the literature so far, are expressed.

\subsection{Likelihood Ratio Test for Separability}

Suppose $\Sigma$ is a spatio-temporal covariance matrix, $\mathbf{U}$ and $\mathbf{V}$ are spatial and temporal covariance matrixes, respectively; testing $H_{0}: \Sigma=\mathbf{U} \otimes \mathbf{V}$ against $H_{1}: \Sigma \neq \mathbf{U} \otimes \mathbf{V}$ is required. Let $n$ be the sample size, $N$ the number of spatial locations, $T$ the number of time locations and $R=N T$. Let

$$
\mathbf{Y}=\mathbf{X B}+\mathbf{E} \Sigma^{\frac{1}{2}}
$$

where $\mathbf{Y}$ is a $n \times R$ observation matrix, $\mathbf{X}$ is a $n \times q$ covariates matrix, $\mathbf{B}=\left(\beta_{1}, \ldots, \beta_{R}\right)$ is a $q \times R$ coefficient matrix, $\mathbf{E}$ is a $R \times m$ matrix with independent rows having $N_{m}(\mathbf{0}, \mathbf{I})$ distribution, and $\Sigma^{\frac{1}{2}}$ is a matrix such that $\Sigma^{\frac{1}{2}} \Sigma^{\frac{1}{2}}=\Sigma$. By earning the maximum likelihood estimator of the parameters 
under the null and alternative hypothesis yields

$$
-2 \log \lambda=\log \left(\frac{|\hat{\mathbf{U}}|^{T}|\hat{\mathbf{V}}|^{N}}{|\mathbf{S}|}\right)^{n}
$$

where

$$
\begin{aligned}
& \mathbf{S}=\frac{1}{r} \mathbf{Y}^{\prime}\left(I_{r}-\mathbf{X}\left(\mathbf{X}^{\prime} \mathbf{X}\right)^{-1} \mathbf{X}\right) \mathbf{Y} \\
& \hat{\mathbf{U}}=\frac{1}{T n} \sum_{k=1}^{n}\left(\mathbf{Y}_{k}-\hat{\mathbf{M}}_{k}\right)^{\prime} \hat{\mathbf{V}}^{-1}\left(\mathbf{Y}_{k}-\hat{\mathbf{M}}_{k}\right), \\
& \hat{\mathbf{V}}=\frac{1}{N n} \sum_{k=1}^{n}\left(\mathbf{Y}_{k}-\hat{\mathbf{M}}_{k}\right) \hat{\mathbf{U}}^{-1}\left(\mathbf{Y}_{k}-\hat{\mathbf{M}}_{k}\right)^{\prime},
\end{aligned}
$$

in which

$$
\mathbf{Y}_{k}=\left(\begin{array}{ccc}
y_{k, 1 \cdot 1} & \ldots & y_{k, 1 \cdot N} \\
\vdots & \ldots & \vdots \\
y_{k, T \cdot 1} & \ldots & y_{k, T \cdot N}
\end{array}\right) \quad \text { and } \quad \mathbf{M}_{k}=E\left(\mathbf{Y}_{k}\right), k=1, \ldots, n
$$

Mitchell et al. (2006) proved that the distribution of $-2 \log \lambda$ under the null hypothesis dose not depend on $\mathbf{B}, \mathbf{U}$ and $\mathbf{V}$. They also showed that the statistic $-2 \log \lambda$ has approximately chi-square distribution with degree of freedom equal to

$$
\frac{N T(N T+1)}{2}-\frac{N(N+1)}{2}-\frac{T(T+1)}{2}+1,
$$

and the empirical critical value is very close to $K \chi_{1-\alpha}^{2}$, where $\chi_{1-\alpha}^{2}$ denotes the $(1-\alpha)$ th quantile of chi-square distribution and

$$
\begin{aligned}
K= & \frac{-n\left[N T \log 2+\sum_{j=1}^{N T} \phi\left(\frac{n-j}{2}\right)-N T \log n\right]}{\frac{N T(N T+1)}{2}-\frac{N(N+1)}{2}-\frac{T(T+1)}{2}+1} \\
& -\frac{\left(\frac{n}{n-1}\right)\left(\frac{N(N+1)}{2}+\frac{T(T+1)}{2}+N T-1\right)}{\frac{N T(N T+1)}{2}-\frac{N(N+1)}{2}-\frac{T(T+1)}{2}+1} .
\end{aligned}
$$




\subsection{Nonparametric Test of Symmetry and Separability}

Considering $\left\{Z(\mathrm{~s}, t): \mathrm{s} \in R^{d}, t \in R\right\}$ to be a spatio-temporal random field, $\Lambda \subseteq R^{d} \times R$ to be a set of spatio-temporal lags, $p$ to be the cardinality of $\Lambda$, $D$ to be the domain of observations, $G=\{C(\mathbf{h}, u):(\mathbf{h}, u) \in \Lambda\}$ to be the set of all covariances on $\Lambda$ and $\hat{G}=\{\hat{C}(\mathbf{h}, u):(\mathbf{h}, u) \in \Lambda\}$ to be the estimator of covariance functions in $G$ over $D$; the null hypothesis of symmetry and separability (2) and (4) can be rewritten as

$$
A f(G)=0,
$$

where $A$ is a matrix of row $\operatorname{rank} q$ and $f$ is a real-valued function differentiable at $G$ takes pairwise ratio of elements in $G$. For example, if

$$
\begin{aligned}
\Lambda= & \left((\mathbf{0}, 0),\left(\mathbf{h}_{1}, u_{1}\right),\left(\mathbf{h}_{1},-u_{1}\right),\left(\mathbf{h}_{2}, u_{2}\right),\left(\mathbf{h}_{2},-u_{2}\right),\left(\mathbf{h}_{1}, 0\right),\left(\mathbf{0}, u_{1}\right),\right. \\
& \left.\left(\mathbf{h}_{2}, 0\right),\left(\mathbf{0}, u_{2}\right)\right)^{\prime},
\end{aligned}
$$

then

$$
\begin{aligned}
G= & \left(C(\mathbf{0}, 0), C\left(\mathbf{h}_{1}, u_{1}\right), C\left(\mathbf{h}_{1},-u_{1}\right), C\left(\mathbf{h}_{2}, u_{2}\right), C\left(\mathbf{h}_{2},-u_{2}\right), C\left(\mathbf{h}_{1}, 0\right), C\left(\mathbf{0}, u_{1}\right),\right. \\
& \left.C\left(\mathbf{h}_{2}, 0\right), C\left(\mathbf{0}, u_{2}\right)\right)^{\prime} .
\end{aligned}
$$

By choosing

$$
\begin{gathered}
f(G)=\left(\frac{C\left(\mathbf{h}_{1}, u_{1}\right)}{C\left(\mathbf{h}_{1}, 0\right)}, \frac{C\left(\mathbf{h}_{1},-u_{1}\right)}{C\left(\mathbf{h}_{1}, 0\right)}, \frac{C\left(\mathbf{h}_{2}, u_{2}\right)}{C\left(\mathbf{h}_{2}, 0\right)}, \frac{C\left(\mathbf{h}_{2},-u_{2}\right)}{C\left(\mathbf{h}_{2}, 0\right)}, \frac{C\left(\mathbf{0}, u_{1}\right)}{C(\mathbf{0}, 0)}, \frac{C\left(\mathbf{0}, u_{2}\right)}{C(\mathbf{0}, 0)}\right)^{\prime}, \\
A_{1}=\left(\begin{array}{ccccccccc}
0 & 1 & -1 & 0 & 0 & 0 & 0 & 0 & 0 \\
0 & 0 & 0 & 1 & -1 & 0 & 0 & 0 & 0
\end{array}\right),
\end{gathered}
$$

and

$$
A_{2}=\left(\begin{array}{cccccc}
1 & 0 & 0 & 0 & -1 & 0 \\
0 & 1 & 0 & 0 & -1 & 0 \\
0 & 0 & 1 & 0 & 0 & -1 \\
0 & 0 & 0 & 1 & 0 & -1
\end{array}\right)
$$

the null hypothesizes of symmetry and separability are equivalent to $A_{1} G=0$ and $A_{2} f(G)=0$, respectively. Considering $\hat{C}_{T}(\mathbf{h}, u)$ as the estimator of $C(\mathbf{h}, u)$ based on observations in a sequence of increasing index sets $D_{T}=$ $S \times \mathcal{T}$ and $\hat{G}_{T}=\left\{\hat{C}_{T}(\mathbf{h}, u):(\mathbf{h}, u) \in \Lambda\right\}$, where $S \subset R^{d_{1}}$ is a fixed space 
at regularly spaced and $\mathcal{T} \subset R^{d_{2}}$ is an increasing space. Li et al. (2007) defined an estimator of $C$ for the set of time $\mathcal{T}=\{1, \ldots, T\}$ under zero mean assumption of $Z$ as follows

$$
\hat{C}_{T}(\mathbf{h}, u)=\frac{1}{|S(\mathbf{h})||\mathcal{T}|} \sum_{\mathbf{s} \in S(\mathbf{h})} \sum_{t=1}^{T-u} Z(\mathbf{s}, t) Z(\mathbf{s}+\mathbf{h}, t+u),
$$

where $S(\mathbf{h})=\{\mathbf{s}: \mathbf{s} \in S, \mathbf{s}+\mathbf{h} \in S\}$ and $|S(\mathbf{h})|$ denote the cardinality of $S(\mathbf{h})$. In addition, consider the mixing coefficient

$$
\alpha(u)=\sup _{A, B}\left\{|P(A \cap B)-P(A) P(B)|, A \in \mathcal{S}_{-\infty}^{0}, B \in \mathcal{S}_{u}^{\infty}\right\},
$$

where $\mathcal{S}_{-\infty}^{0}$ is a $\sigma$-algebra generated by past process until $t=0$ that satisfies strong mixing condition

$$
\alpha(u)=O\left(u^{-\varepsilon}\right),
$$

and assuming that the moment condition for $\hat{C}_{T}(\mathbf{h}, u)$ is given by

$$
\sup _{T} E\left\{\left|\sqrt{|\mathcal{T}|}\left\{\hat{C}_{T}(\mathbf{h}, u)-C(\mathbf{h}, u)\right\}\right|^{2+\delta}\right\} \leqslant C_{\delta} ; \quad \delta>0, C_{\delta}<\infty .
$$

Theorem 1. (Li et al., 2007) Let $\left\{Z(s, t): s \in R^{d}, t \in R\right\}$ be a strictly stationary spatio-temporal random field on $D_{T}=S \times \mathcal{T}$ and conditions (10) and (11) hold. Then the test statistics for symmetry and separability hypothesis are respectively given by

$$
\begin{aligned}
T S 1 & =|\mathcal{T}|\left\{A_{1} \hat{G}_{T}\right\}^{\prime}\left(A_{1} \Sigma A_{1}^{\prime}\right)^{-1}\left\{A_{1} \hat{G}_{T}\right\} \longrightarrow \chi_{q_{1}}^{2}, \\
T S 2 & =|\mathcal{T}|\left\{A_{2} f\left(\hat{G}_{T}\right)\right\}^{\prime}\left(A_{2} B^{\prime} \Sigma B A_{2}^{\prime}\right)^{-1}\left\{A_{2} f\left(\hat{G}_{T}\right)\right\} \longrightarrow \chi_{q_{2}}^{2},
\end{aligned}
$$

where $r$ is the element numbers of the vector $f(G), B_{i j}=\frac{\partial f_{i}}{\partial G_{j}}, i=1, \ldots, m, j=$ $1, \ldots, r, q_{1}$ and $q_{2}$ are row ranks of $A_{1}$ and $A_{2}$, respectively.

The matrix $\Sigma$ for separability testing of covariance function based on (12) is unknown and should be estimated. For this purpose, let $m=\max \{|u|$ : $(\mathbf{h}, u) \in \Lambda\}, T_{m}=T-m$. Shao and $\operatorname{Li}(2009)$ define the recursive estimator of $C(\mathbf{h}, u)$ based on the temporal subsampling of $\{Z(\mathbf{s}, t): \mathbf{s} \in S, t=1, \ldots, J+$ $m\}$ as

$$
\widetilde{C}_{J}(\mathbf{h}, u)=\frac{\sum_{\mathbf{s} \in S(\mathbf{h})} \sum_{t=1}^{J} Z(\mathbf{s}, t) Z(\mathbf{s}+\mathbf{h}, t+u)}{|S(\mathbf{h})||J|} ; \quad J=1, \ldots, T_{m} .
$$


Let $\widetilde{G}_{J}=\left\{\widetilde{C}_{J}(\mathbf{h}, u),(\mathbf{h}, u) \in \Lambda\right\}$, then the first test statistic can be written as

$$
T S_{1}=T\left\{A f\left(\widetilde{G}_{T_{m}}\right)\right\}^{\prime}\left(A \widetilde{B}^{\prime} \widetilde{\Sigma} \widetilde{B} A^{\prime}\right)^{-1}\left\{A f\left(\widetilde{G}_{T_{m}}\right)\right\}
$$

where $\widetilde{B}=B\left(\widetilde{G}_{T_{m}}\right)$ and $\widetilde{\Sigma}=N^{-2} \sum_{J=1}^{T_{m}} J^{2}\left(\widetilde{G}_{J}-\widetilde{G}_{T_{m}}\right)\left(\widetilde{G}_{J}-\widetilde{G}_{T_{m}}\right)^{T}$. The test statistic $T S_{2}$ can also be redefined as

$$
T S_{2}=T\left\{A f\left(\widetilde{G}_{T_{m}}\right)\right\}^{\prime} \widetilde{V}_{N}^{-1}\left\{A f\left(\widetilde{G}_{T_{m}}\right)\right\}
$$

where $\widetilde{V}_{N}=N^{-2} \sum_{J=1}^{T_{m}} J^{2}\left(A f\left(\widetilde{G}_{J}\right)-A f\left(\widetilde{G}_{T_{m}}\right)\right)\left(A f\left(\widetilde{G}_{J}\right)-A f\left(\widetilde{G}_{T_{m}}\right)\right)^{\prime}$. Shao and $\mathrm{Li}$ (2009) assert that $T S_{1}$ and $T S_{2}$ are asymptotically equivalent to a random variable $U_{q}$, that its $\alpha$ th quantiles have been tabulated in Lobato (2001) for $q=1, \ldots, 20$.

\subsection{Spectral Test of Separability}

Let $\left\{Z(\mathbf{s}, t): \mathbf{s} \in D \in R^{d}, t \in Z\right\}$ be a zero mean stationary spatio-temporal random field observed at $N \times T$ space-time sites. By the Bochner's theorem the spectral density $g$ yields from the inverse Fourier transformation as

$$
\begin{aligned}
g(\omega, \tau) & =\frac{1}{(2 \pi)^{d+1}} \iint \exp \left\{-i \omega^{\prime} \mathbf{h}-i \tau u\right\} C(\mathbf{h}, u) d \mathbf{h} d u ; \quad(\mathbf{h}, u) \in R^{d} \times R \\
& =\frac{1}{(2 \pi)^{d}} \int \exp \left\{-i \omega^{\prime} \mathbf{h}\right\} f(\mathbf{h}, \tau) d h
\end{aligned}
$$

where $f(\mathbf{h}, \tau)$, the cross-spectral density function of $Y_{1}(t)=Z(\mathbf{s}, t)$ and $Y_{2}(t)=Z(\mathbf{s}+\mathbf{h}, t)$, is defined as

$$
f(\mathbf{h}, \tau)=(2 \pi)^{-1} \sum_{u=-\infty}^{\infty} \exp \{-i u \tau\} C(\mathbf{h}, u) ; \quad \tau \in[0, \infty) .
$$

If the covariance function $C(\mathbf{h}, u)$ is separable, then $f(\mathbf{h}, \tau)$ can be decomposed as product of two functions of $\mathbf{h}$ and $\tau$ as follow

$$
f(\mathbf{h}, \tau)=(2 \pi)^{-1} \sum_{u=-\infty}^{\infty} \exp \{-i u \tau\} C_{S}(\mathbf{h}) C_{T}(u)=C_{S}(\mathbf{h}) \kappa(\tau) .
$$

When the random field $Z(\mathbf{s}, t)$ is not spatially stationary, for any two spatial locations $\mathbf{a}, \mathbf{b} \in D$, the cross-spectral density function of $Y_{1}(t)=Z(\mathbf{a}, t)$ and 
$Y_{2}(t)=Z(\mathbf{b}, t)$ depends on $\mathbf{a}$ and $\mathbf{b}$ and under the separability assumption of the covariance function is given by

$$
f_{\mathbf{a b}}(\tau)=(2 \pi)^{-1} \sum_{u=-\infty}^{\infty} \exp \{-i u \tau\} C(Z(\mathbf{a}, t), Z(\mathbf{b}, t+u))=C_{S}(\mathbf{a}, \mathbf{b}) \cdot C_{T}(u) .
$$

The coherency between two locations $\mathbf{a}$ and $\mathbf{b}$ is defined by

$$
R_{\mathrm{ab}}(\tau)=\frac{\left|f_{\mathrm{ab}}(\tau)\right|}{\left[f_{\mathrm{aa}}(\tau) \cdot f_{\mathrm{bb}}(\tau)\right]^{\frac{1}{2}}} .
$$

The separability assumption of covariance function yields

$$
R_{\mathrm{ab}}(\tau)=\frac{\left|C_{S}(\mathbf{a}, \mathbf{b})\right|}{\left[C_{S}(\mathbf{a}, \mathbf{a}) C_{S}(\mathbf{b}, \mathbf{b})\right]^{\frac{1}{2}}} .
$$

Therefore, the coherency dose not depend on the frequency $\tau$ when the random field is separable. So, the test of separability can be done by studying if $R_{\mathrm{ab}}(\tau)$ depends on the frequency of $\tau$ or not. To estimate the cross-spectral density $f_{\mathbf{a}, \mathbf{b}}$, first consider the taper Fourier transform defined by

$$
J_{\mathbf{a}}(\omega)=\sum_{t=0}^{T-1} K\left(\frac{t}{T}\right) Z(\mathbf{a}, t) \exp \{-i t \omega\},
$$

where $0<K(t)<1$ is a tapering function. Fuentes (2006) defined a smooth estimator for $f_{\mathbf{a b}}(\tau)$ as

$$
\hat{f}_{\mathbf{a b}}(\omega)=\iint g_{\rho}(\mathbf{a}-\mathbf{s}) g_{\rho}(\mathbf{b}-\mathbf{s}) I_{\mathbf{a}+\mathbf{s}, \mathbf{b}+\mathbf{s}}^{*}(\omega) d \mathbf{s},
$$

where

$$
I_{\mathbf{a b}}^{*}(\omega)=\frac{2 \pi}{T} \sum_{t=0}^{T-1} W^{(T)}\left(\omega-\frac{2 \pi t}{T}\right) I_{\mathbf{a b}}\left(\frac{2 \pi t}{T}\right),
$$

and the weight function $g_{\rho}$ depends on the bandwidth parameter $\rho$ satisfying:

A.1. $g_{\rho}(\mathbf{s}) \geqslant 0$, for all $\mathbf{s}, \rho$.

A.2. $g_{\rho}(\mathbf{s})$ decays to zero as $|\mathbf{s}| \longrightarrow \infty$, for all $\rho$.

A.3. $\int_{-\infty}^{\infty} \int_{-\infty}^{\infty} g_{\rho}(\mathbf{s}) d \mathbf{s}=1$. 
A.4. $\int_{-\infty}^{\infty} \int_{-\infty}^{\infty}\left\{g_{\rho}(\mathbf{s})\right\}^{2} d \mathbf{s}<\infty$.

The cross-periodogram $I_{\mathrm{ab}}(\omega)$ and weight function $W(\alpha)$ in $(15)$ are defined respectively as

$$
I_{\mathbf{a b}}(\omega)=\left[2 \pi \sum_{t=0}^{T-1} K^{2}\left(\frac{t}{T}\right)\right]^{-1} J_{\mathbf{a}}(\omega) J_{\mathbf{b}}^{c}(\omega),
$$

and

$$
W^{(T)}(\alpha)=\frac{1}{B_{T}} \sum_{j=-\infty}^{\infty} W\left\{\frac{(\alpha+2 \pi j)}{B_{T}}\right\},
$$

where $B_{T}$ is a bandwidth parameter and satisfies $\int_{-\infty}^{\infty} W(\alpha) d \alpha=1$. Now an estimator of $R_{\mathrm{ab}}(\tau)$ based on $\hat{f}_{\mathrm{ab}}(\tau)$ is given by $\hat{R}_{\mathrm{ab}}(\tau)=\frac{\left|\hat{f}_{\mathrm{ab}}(\tau)\right|}{\left[\hat{f}_{\mathrm{aa}}(\tau) \hat{f}_{\mathrm{bb}}(\tau)\right]^{\frac{1}{2}}}$. Let $\hat{f}_{D}(\tau)$ be a matrix with entries $\hat{f}_{\mathbf{a b}}(\tau)$ for all pairs $\{\mathbf{a}, \mathbf{b}\}$ in spatial domain D.

Theorem 2. (Fuentes, 2006) Let the assumptions A.1-A.3 hold and the spectral estimators $\hat{f}_{D}\left(\tau_{j}\right), j=1, \ldots, J$ have asymptotic normal distribution, then

B.1. The weight function $W(\beta),-\infty<\beta<\infty$, is real-valued, even, and of bounded variation $\int_{-\infty}^{\infty}|W(\beta)| d \beta<\infty$ and $\int_{-\infty}^{\infty} W(\beta) d \beta=1$,

B.2. $\sum_{u=-\infty}^{\infty}|u||C(\boldsymbol{h}, u)|<\infty$ and $\sum_{u=-\infty}^{\infty}|C(\boldsymbol{h}, u)|<\infty$,

B.3. If $T \rightarrow \infty$ then $B_{T} T \rightarrow \infty$, and $B_{T} \rightarrow 0$.

Using Theorem 2 one can conclude that $\hat{f}_{\mathbf{a}_{1}, \mathbf{b}_{1}(\omega)}$ and $\hat{f}_{\mathbf{a}_{2}, \mathbf{b}_{2}(\tau)}$ are approximately independent if one of the following conditions hold:

C.1. The distance between $\left(\mathbf{a}_{i}, \mathbf{b}_{i}\right)$ and $\left(\mathbf{a}_{j}, \mathbf{b}_{j}\right)$ is greater than the bandwidth of the function $g_{\rho}(\mathbf{u})$.

C.2. $\int_{-\infty}^{\infty} \int_{-\infty}^{\infty}|W(\theta+\omega)|^{2}|W(\theta+\tau)|^{2} d \theta=0$, i.e. $\|\omega \pm \lambda\|$ is bigger than the bandwidth of $|W(\theta)|^{2}$.

Since the variance of $\hat{R}_{\mathbf{a b}}(\tau)$ depends on $\mathbf{a}$ and $\mathbf{b}$, Fuentes (2006) suggested a variance stability transformation of $\hat{R}_{\mathbf{a b}}(\tau)$ as $\hat{\phi}_{\mathbf{a b}}(\tau)=\tanh ^{-1}$ $\left[\hat{R}_{\mathbf{a b}}(\tau)\right]$. Let $\hat{\phi}_{D}(\tau)$ be the matrix with entries $s \hat{\phi}_{\mathbf{a b}}(\tau)$ for all pairs $\{\mathbf{a}, \mathbf{b}\}$ in the spatial domain $D$. Fuentes (2006) showed under assumptions A.1-A.3 and B.1-B.3 that the variables $\hat{\phi}_{D}\left(\tau_{j}\right), j=1, \ldots, J$ have asymptotic normal 
distribution. Consider a set of frequencies $\tau_{j}, j=1, \ldots, J$ and $k$ pairs of locations $\left\{\mathbf{a}_{i}, \mathbf{b}_{i}\right\}$ that cover the domain $D$. We can decompose $\hat{\phi}_{\mathbf{a}, \mathbf{b}}(\tau)$ as

$$
\hat{\phi}_{\mathbf{a}_{i}, \mathbf{b}_{i}}\left(\tau_{j}\right)=\phi_{\mathbf{a}_{i}, \mathbf{b}_{i}}\left(\tau_{j}\right)+\varepsilon\left(\left(\mathbf{a}_{i}, \mathbf{b}_{i}\right), \tau_{j}\right) ; \quad i=1, \ldots, k, j=1, \ldots, J,
$$

where the random variables $\varepsilon_{i j}=\varepsilon\left(\left(\mathbf{a}_{i}, \mathbf{b}_{i}\right), \tau_{j}\right)$ asymptotically satisfy in the following conditions:

D.1. $E\left(\varepsilon_{i j}\right)=0$,

D.2. $\quad \operatorname{var}\left(\varepsilon_{i j}\right)=\sigma^{2} ; \quad i=1, \ldots, k, \quad j=1, \ldots, J$,

D.3. For any of the two locations $\left\{\mathbf{a}_{i}, \mathbf{b}_{i}\right\}$ and $\left\{\mathbf{a}_{k}, \mathbf{b}_{k}\right\}$, and also frequencies of $\tau_{j}$ and $\tau_{l}$ that satisfy the conditions C.1 and C.2, the $\operatorname{cov}\left\{\varepsilon\left(\mathbf{a}_{i}, \mathbf{b}_{i}\right)\left(\tau_{j}\right), \varepsilon\left(\mathbf{a}_{k}, \mathbf{b}_{k}\right)\left(\tau_{l}\right)\right\}$ is equal to zero.

Setting $U_{i j}=\hat{\phi}_{\mathbf{a}_{i} \mathbf{b}_{i}}\left(\tau_{j}\right)$ and $m_{i j}=\phi_{\mathbf{a}_{i}, \mathbf{b}_{i}}\left(\tau_{j}\right)$, then we have the model

$$
U_{i j}=m_{i j}+\varepsilon_{i j} ; \quad i=1, \ldots, k, j=1, \ldots, J,
$$

that becomes a two factor analysis of variance model given by

$$
U_{i j}=\mu+\alpha_{i}+\beta_{j}+\varepsilon_{i j} ; \quad i=1, \ldots, k, j=1, \ldots, J .
$$

The parameters $\alpha_{i}$ and $\beta_{j}$ represent the main effects of the spatial and frequency factors. Separability of the covariance function requires the coherency function to be independent of the frequency factor, so the separability test of the covariance function is equivalent to the two factor analysis of the model (16) when $\beta_{j}$ is equal to zero. Therefore the hypothesis of the separability test can be written as

$$
\left\{\begin{array}{l}
H_{0}: U_{i j}=\mu+\alpha_{i}+\varepsilon_{i j} \\
H_{1}: U_{i j}=\mu+\alpha_{i}+\beta_{j}+\varepsilon_{i j}
\end{array}\right.
$$

\subsection{Spectral Test for Symmetry}

In general, the cross-spectral density function $f_{\mathbf{a b}}(\tau)$ in (14) is a complicated function, but under the axial symmetry in time, it is real-valued. The phase between $Z(\mathbf{a}, \cdot)$ and $Z(\mathbf{b}, \cdot)$ defined by

$$
\phi_{\mathbf{a b}}(\tau)=\tan ^{-1}\left\{\frac{\operatorname{Im} f_{\mathrm{ab}}(\tau)}{\operatorname{Re} f_{\mathrm{ab}}(\tau)}\right\},
$$

is zero under axial symmetry in time. Based on (15), this function is estimated by $\hat{\phi}_{\mathbf{a b}}(\tau)=\tan ^{-1}\left\{\frac{\operatorname{Im} \hat{f}_{\mathrm{ab}}(\tau)}{\operatorname{Re} \hat{f}_{\mathrm{ab}}(\tau)}\right\}$. Park and Fuentes (2008) considered 
that under the assumptions B.1-B.3, the estimator $\hat{\phi}_{\mathbf{a b}}(\tau)$ has asymptotic normal distribution with mean 0 and asymptotic variance

$$
\lim _{T \rightarrow \infty} B_{T} T \operatorname{var}\left(\hat{\phi}_{\mathbf{a b}}(\lambda)\right)=\pi \int_{R} W_{T}^{2}(\alpha) d \alpha(1-\eta(2 \lambda))\left[\left|R_{\mathbf{a b}}(\lambda)\right|^{-2}-1\right] .
$$

Since the variance of $\hat{\phi}_{\mathbf{a b}}(\tau)$ depends on $\mathbf{a}$ and $\mathbf{b}$, an appropriate transformation to stabilize the asymptotic variance is given by

$$
\hat{\phi}_{\mathbf{a b}}^{*}(\tau)=\frac{\hat{\phi}_{\mathbf{a b}}(\tau)}{\left[\left|\hat{R}_{\mathbf{a b}}(\tau)\right|^{-2}-1\right]^{\frac{1}{2}}},
$$

which has asymptotic normal distribution. Consider a set of $J$ frequencies $\tau_{j} ; j=1, \ldots, J$ and $k$ pairs of locations $\left\{\left(\mathbf{a}_{i}, \mathbf{b}_{i}\right)\right\}_{i=1}^{k}$, that covers the spatial domain satisfying the conditions C.1 or C.2 and $\mathbf{a}_{i}-\mathbf{b}_{i}=\mathbf{h}$. The estimator $\hat{\phi}_{\mathbf{a b}}^{*}\left(\tau_{j}\right)$ can be decomposed as

$$
\hat{\phi}_{\mathbf{a}_{i} \mathbf{b}_{i}}^{*}\left(\tau_{j}\right)=\phi_{\mathbf{a}_{i} \mathbf{b}_{i}}^{*}\left(\tau_{j}\right)+\epsilon\left(\left(\mathbf{a}_{i}, \mathbf{b}_{i}\right), \tau_{j}\right) ; \quad i=1, \ldots, k, j=1, \ldots, J,
$$

where the variables $\epsilon\left(\left(\mathbf{a}_{i} \mathbf{b}_{i}\right), \tau_{j}\right)$ asymptotically satisfy the conditions D.1D.3. The model (17) can be expressed as

$$
U_{i j}=\mu+\alpha_{i}+\beta_{j}+\varepsilon_{i j} ; \quad i=1, \ldots, k, j=1, \ldots, J,
$$

where the parameters $\alpha_{i}$ and $\beta_{j}$ denote the spatial and temporal effects, respectively. Since under the axial symmetry in time the phase is zero, then the temporal frequency factor would also be zero. Therefore the testing hypothesis for axial symmetry in time is given by

$$
\left\{\begin{array}{l}
H_{0}: U_{i j}=\mu+\alpha_{i}+\varepsilon_{i j} \\
H_{1}: U_{i j}=\mu+\alpha_{i}+\beta_{j}+\varepsilon_{i j}
\end{array} .\right.
$$

The spectral density function (13) can be expressed as

$$
g(\omega, \tau)=\frac{1}{(2 \pi)^{2}} \int_{R} \int_{R} \exp \left\{-i h_{2} \omega_{2}-i u \tau\right\} k\left(\omega_{1}, h_{2}, u\right) d h_{2} d u,
$$

where $k\left(\omega_{1}, h_{2}, u\right)$ is cross-spectral density function of $Z\left(\cdot, a_{2}, t\right)$ and $Z\left(\cdot, a_{2}+\right.$ $\left.h_{2}, t+u\right)$ defined by

$$
k\left(\omega_{1}, h_{2}, u\right)=\frac{1}{(2 \pi)} \int_{R} \exp \left\{-i h_{1} \omega_{1}\right\} C(\mathbf{h}, u) d h_{1} .
$$


Under the spatial axially symmetry, $\psi\left(\omega_{1}, h_{2}, u\right)=0$. An estimation of the phase function is given by $\hat{\psi}\left(\omega_{1}, h_{2}, u\right)=\tan ^{-1}\left\{\frac{\operatorname{Im} \hat{k}\left(\omega_{1}, h_{2}, u\right)}{\operatorname{Re} \hat{k}\left(\omega_{1}, h_{2}, u\right)}\right\}$, where

$$
\hat{I}^{*}{ }_{\Delta_{1}}\left(\omega_{1}, h_{2}, u\right)=\frac{2 \pi}{N_{1}} \sum_{n_{1}=1}^{N_{1}-1} \hat{W}_{s}\left(\omega_{1}-\frac{2 \pi n_{1}}{N_{1}}\right) \hat{I}_{\Delta_{1}}^{s}\left(\frac{2 \pi n_{1}}{N_{1}}, h_{2}, u\right),
$$

$\Delta_{1}$ is the unit distance of the first spatial coordinate and

$$
\begin{aligned}
\hat{I}_{\Delta_{1}}^{s}\left(\omega_{1}, h_{2}, u\right)= & {\left[2 \pi \sum_{n_{1}=1}^{N_{1}-1} K^{2}\left(\frac{n_{1}}{N_{1}}\right) \Delta_{1}\right]^{-1} J_{\Delta_{1}}\left(\omega_{1}, a_{2}, t\right) } \\
& \times J_{\Delta_{1}}^{c}\left(\omega_{1}, a_{2}+h_{2}, t+u\right) .
\end{aligned}
$$

Since $\operatorname{var}(\hat{\psi})$ depends on $h_{2}$ and $u$, Park and Fuentes (2008) suggested a variance stability transformation as

$$
\hat{\psi}_{\Delta_{1}}^{*}\left(\omega_{1}, h_{2}, u\right)=\frac{\hat{\psi}_{\Delta_{1}}\left(\omega_{1}, h_{2}, u\right)}{\left[\left.\hat{Q}_{\Delta_{1}}\left(\omega_{1}, h_{2}, u\right)\right|^{-2}-1\right]^{\frac{1}{2}}} .
$$

Consider a set of $J$ frequencies $\omega_{j} ; j=1, \ldots, J$ and $k$ pairs of $\left\{\left(a_{2}^{i}, t^{\mathbf{a}_{i}}\right)\right.$, $\left.\left(b_{2}^{i}, t^{\mathbf{b}_{i}}\right)\right\}$, where $a_{2}^{i}$ and $b_{2}^{i}$ are the second spatial coordinate, and $t^{\mathbf{a}_{i}}$ and $t^{\mathbf{b}_{i}}$ are temporal locations. The selected pairs cover the domain and satisfy the condition C.1 or C.2, and $b_{2}^{i}-a_{2}^{i}=h_{2}, t^{\mathbf{b}_{i}}-t^{\mathbf{a}_{i}}=u$. The estimator $\hat{\psi}_{\Delta_{1}}^{*}\left(\omega_{j}, b_{2}^{i}-a_{2}^{i}, t^{\mathbf{b}_{i}}-t^{\mathbf{a}_{i}}\right)=\hat{\psi}_{i}^{*}\left(\omega_{j}\right)$ can be decomposed as (18), where the parameters $\alpha_{i}$ and $\beta_{j}$ are spatio-temporal interaction effect and the spectral frequency effect, respectively. Since under spatial axially symmetry phase is zero, the spectral frequency effect is also zero. So the testing hypothesis for spatial axial symmetry is given by

$$
\left\{\begin{array}{l}
H_{0}: U_{i j}=\mu+\alpha_{i}+\varepsilon_{i j} \\
H_{1}: U_{i j}=\mu+\alpha_{i}+\beta_{j}+\varepsilon_{i j}
\end{array}\right.
$$

\section{Evaluation of the Separability Tests}

In this section the separability testing hypothesizes proposed by Mitchell (2006), Li et al. (2007), Shao and Li (2009) and Fuentes (2006) are evaluated and compared under different conditions. First the Mitchell's test is considered at the spatial locations $(1,1),(1,2),(1,3)$ on a $3 \times 3$ grid. For testing 
$\Sigma=\mathbf{U} \otimes \mathbf{V}$, where $\mathbf{U}$ and $\mathbf{V}$ are $3 \times 3$ spatial and $T \times T$ temporal covariance matrices, respectively, based on Theorem 1, without loss of generality, we chose $\mathbf{B}=\left(\mu_{11}, \ldots, \mu_{1 T}, \ldots, \mu_{31}, \ldots, \mu_{3 T}\right)=\mathbf{0}$ and regarding that $\mathbf{X}=1_{R}$, where $1_{R}$ is $R \times 1$ vector of ones. To evaluate this test based on empirical size, the separable covariance function

$$
C(\mathbf{h}, u)=\exp \left(-a^{2}\|\mathbf{h}\|\right)\left\{1+\left(1+\frac{u^{2}}{b^{2}}\right)^{-\beta}\right\},
$$

is considered, where $a$ and $b$ are spatial and temporal scale coefficients whilst $\beta$ is the shape parameter. The estimates $\hat{\mathbf{U}}$ and $\hat{\mathbf{V}}$ in (6) are computed using a "lip-flop" scheme by choosing $\mathbf{V}=I_{T}$ as initial value. To evaluate the test based on empirical power, the nonseparable covariance function

$$
C(\mathbf{h}, u)=\sigma_{0}^{2} I_{\{(\mathbf{h}, u)=0\}}(\mathbf{h}, u)+\sigma^{2} \exp \left(\frac{\|\mathbf{h}\|+u^{2}}{a}\right)^{\frac{1}{2}},
$$

is considered, where $I_{\{\cdot\}}$ is an indicator function and $\sigma_{0}^{2}=0.01$ is the nugget effect, $\alpha=2$ is the range and $\sigma^{2}=1$ is the partial sill. For different values of $a, b, \beta$ and $T$ the empirical sizes and powers are computed by 100 iterated simulations. The nominal level is set to be 0.05 and critical values are chosen from Mitchell's tables (Mitchell, 2006). Table 1 shows the smaller values of $T$ correspond to the smaller test sizes, and also changes of $a, b$ and $\beta$ have no affect on the size of separable tests. But when $T$ is large and $n$ takes small values the size of test is close to 1 . Therefore the Mitchell test is not suitable for some values of $a, b$ and $\beta$. For example for $\beta=0.1, T=40, a=0.1$ and $b=2$ when $n \leqslant 1000$ this test has not acceptable empirical sizes, even when $T=50, a=0.1$ and $b=2$ this test is not useful for $n \leqslant 5000$. For large values of $T$, the empirical size increases as $a, b$ and $\beta$ increase. Since the Mitchell test of separability is only valid for $n>3 T$, for different fixed values of $T$, the empirical powers of this test, in terms of the smallest possible values of $n$, are shown in Table 3 . As one can see the empirical power of the test increases when either $n$ or $T$ increases. However this test is only acceptable for $T \geqslant 50$ and appropriate values of $\Lambda$.

To compare the Fuentes test with Shao and Li test of separability, the function $W(\alpha)$ with a bandwidth of $2 \pi B_{T}, B_{T}=\frac{1}{12}, g_{\rho}(\mathbf{s})=g_{1}(s) . g_{2}(s)$, $\rho=5.5$ is given by

$$
W_{(\mathbf{a b})}^{(T)}\left(\frac{2 \pi s}{T}\right)=\frac{T}{2 \pi}(2 m+1)^{-1},
$$


and

$$
g_{1}(s)=g_{2}(s)=\left\{\begin{array}{cc}
\frac{1}{\rho} & \frac{-\rho}{2} \leqslant s \leqslant \frac{-\rho}{2} \\
0 & \text { O. W. }
\end{array}\right.
$$

In order to obtain approximately uncorrelated estimates, each frequency $\omega_{j}$ was chosen as $\omega_{j}=\frac{\pi(3(j-1)+1)}{14}$ with distance of $\frac{3 \pi}{14}$ witch exceeds $\frac{\pi}{6}$. The pairs of $\left\{\left(a_{i}, b_{i}\right)\right\}_{i=1}^{n}$ were chosen in a $30 \times 30$ spatial regular grid so that the grid cells distances are at least 5.5. We evaluate $\hat{f}_{\mathbf{a b}(\omega)}$ at the frequencies,

$$
\omega_{1}=\frac{\pi}{14}, \quad \omega_{2}=\frac{4 \pi}{14}, \quad \omega_{3}=\frac{7 \pi}{14}, \quad \omega_{4}=\frac{10 \pi}{14}, \quad \omega_{5}=\frac{13 \pi}{14},
$$

\begin{tabular}{|c|c|c|c|c|c|c|c|c|c|c|c|}
\hline \multirow{2}{*}{$\mathrm{T}$} & \multirow{2}{*}{$n$} & \multirow{2}{*}{$\begin{array}{l}a \\
b\end{array}$} & \multicolumn{3}{|c|}{0.1} & \multicolumn{3}{|c|}{1} & \multicolumn{3}{|c|}{2} \\
\hline & & & 0.1 & 1 & 2 & 0.1 & 1 & 2 & 0.1 & 1 & 2 \\
\hline \multirow{5}{*}{5} & 20 & & 5 & 5 & 8 & 6 & 5 & 3 & 6 & 5 & 7 \\
\hline & 50 & & 10 & 6 & 0 & 5 & 4 & 7 & 4 & 6 & 6 \\
\hline & 100 & & 7 & 9 & 8 & 3 & 9 & 4 & 7 & 3 & 3 \\
\hline & 300 & & 5 & 4 & 8 & 3 & 3 & 3 & 3 & 3 & 3 \\
\hline & 500 & & 5 & 4 & 7 & 5 & 7 & 4 & 3 & 8 & 4 \\
\hline \multirow{5}{*}{10} & 50 & & 5 & 4 & 8 & 5 & 3 & 6 & 5 & 9 & 6 \\
\hline & 100 & & 4 & 2 & 3 & 8 & 7 & 6 & 2 & 9 & 5 \\
\hline & 300 & & 2 & 3 & 4 & 4 & 5 & 2 & 7 & 3 & 5 \\
\hline & 500 & & 4 & 5 & 4 & 7 & 1 & 7 & 5 & 9 & 8 \\
\hline & 1000 & & 4 & 5 & 1 & 6 & 6 & 1 & 5 & 3 & 5 \\
\hline \multirow{5}{*}{40} & 200 & & 6 & 4 & 100 & 3 & 5 & 4 & 1 & 1 & 5 \\
\hline & 500 & & 4 & 3 & 100 & 7 & 5 & 7 & 6 & 4 & 3 \\
\hline & 1000 & & 2 & 5 & 41 & 6 & 6 & 5 & 2 & 6 & 8 \\
\hline & 2000 & & 4 & 3 & 5 & 3 & 3 & 4 & 3 & 4 & 4 \\
\hline & 3000 & & 8 & 6 & 4 & 6 & 9 & 2 & 0 & 3 & 6 \\
\hline \multirow{5}{*}{50} & 200 & & 6 & 100 & 100 & 1 & 5 & 3 & 4 & 7 & 7 \\
\hline & 500 & & 3 & 2 & 100 & 0 & 2 & 3 & 6 & 5 & 3 \\
\hline & 1000 & & 3 & 2 & 100 & 3 & 6 & 3 & 3 & 8 & 8 \\
\hline & 2000 & & 6 & 6 & 100 & 5 & 7 & 3 & 3 & 5 & 6 \\
\hline & 3000 & & 8 & 3 & 100 & 1 & 4 & 2 & 3 & 2 & 3 \\
\hline \multirow{5}{*}{100} & 400 & & 100 & 100 & 100 & 1 & 100 & 100 & 2 & 100 & 100 \\
\hline & 500 & & 100 & 100 & 100 & 3 & 100 & 100 & 1 & 20 & 100 \\
\hline & 1000 & & 100 & 100 & 100 & 3 & 1 & 100 & 4 & 5 & 100 \\
\hline & 2000 & & 100 & 100 & 100 & 2 & 5 & 100 & 4 & 6 & 100 \\
\hline & 3000 & & 100 & 100 & 100 & 7 & 6 & 100 & 4 & 4 & 100 \\
\hline
\end{tabular}

Table 1. Empirical sizes $(\times 100)$ of Mitchell separability test for $\beta=0.1$ 
and at the following 6 pairs of spatial locations:

$$
\begin{array}{ll}
\mathbf{a}_{1}=(1,1) & \mathbf{b}_{1}=(1,2) \\
\mathbf{a}_{2}=(9,1) & \mathbf{b}_{2}=(2,2) \\
\mathbf{a}_{3}=(17,1) & \mathbf{b}_{3}=(17,2) \\
\mathbf{a}_{4}=(1,10) & \mathbf{b}_{4}=(1,11) \\
\mathbf{a}_{5}=(9,10) & \mathbf{b}_{5}=(9,11) \\
\mathbf{a}_{6}=(17,10) & \mathbf{b}_{6}=(17,11) .
\end{array}
$$

\begin{tabular}{|c|c|c|c|c|c|c|c|c|c|c|c|}
\hline \multirow{2}{*}{$\mathrm{T}$} & \multirow{2}{*}{$n$} & \multicolumn{2}{|l|}{$a$} & \multicolumn{2}{|l|}{0.1} & \multicolumn{3}{|c|}{1} & \multicolumn{3}{|c|}{2} \\
\hline & & $b$ & 0.1 & 1 & 2 & 0.1 & 1 & 2 & 0.1 & 1 & 2 \\
\hline \multirow{5}{*}{5} & 20 & & 6 & 7 & 5 & 7 & 8 & 12 & 5 & 8 & 4 \\
\hline & 50 & & 7 & 3 & 8 & 4 & 8 & 5 & 5 & 6 & 3 \\
\hline & 100 & & 3 & 7 & 6 & 3 & 8 & 7 & 5 & 6 & 6 \\
\hline & 300 & & 3 & 9 & 4 & 6 & 4 & 1 & 9 & 5 & 5 \\
\hline & 500 & & 4 & 7 & 3 & 2 & 5 & 8 & 2 & 10 & 10 \\
\hline \multirow{5}{*}{10} & 50 & & 7 & 5 & 3 & 3 & 5 & 2 & 4 & 2 & 5 \\
\hline & 100 & & 7 & 4 & 4 & 2 & 8 & 5 & 8 & 7 & 10 \\
\hline & 300 & & 6 & 6 & 5 & 2 & 2 & 3 & 6 & 3 & 7 \\
\hline & 500 & & 5 & 2 & 5 & 6 & 3 & 3 & 4 & 6 & 10 \\
\hline & 1000 & & 5 & 3 & 9 & 5 & 2 & 3 & 4 & 3 & 5 \\
\hline \multirow{5}{*}{40} & 200 & & 6 & 3 & 8 & 10 & 3 & 0 & 6 & 6 & 3 \\
\hline & 500 & & 7 & 8 & 8 & 5 & 2 & 11 & 5 & 3 & 4 \\
\hline & 1000 & & 5 & 4 & 8 & 6 & 8 & 5 & 4 & 7 & 2 \\
\hline & 2000 & & 6 & 4 & 8 & 6 & 6 & 2 & 3 & 5 & 4 \\
\hline & 3000 & & 4 & 6 & 8 & 2 & 4 & 5 & 1 & 5 & 9 \\
\hline \multirow{5}{*}{50} & 200 & & 5 & 6 & 5 & 6 & 4 & 4 & 3 & 5 & 4 \\
\hline & 500 & & 4 & 1 & 2 & 2 & 3 & 4 & 2 & 7 & 0 \\
\hline & 1000 & & 5 & 3 & 5 & 5 & 7 & 6 & 6 & 5 & 5 \\
\hline & 2000 & & 3 & 8 & 6 & 8 & 4 & 3 & 5 & 4 & 4 \\
\hline & 3000 & & 7 & 6 & 4 & 1 & 7 & 1 & 5 & 5 & 4 \\
\hline \multirow{5}{*}{100} & 400 & & 100 & 100 & 100 & 0 & 4 & 2 & 2 & 4 & 1 \\
\hline & 500 & & 100 & 100 & 100 & 1 & 1 & 1 & 3 & 3 & 5 \\
\hline & 1000 & & 100 & 100 & 100 & 0 & 0 & 3 & 1 & 3 & 1 \\
\hline & 2000 & & 100 & 100 & 100 & 3 & 1 & 6 & 7 & 1 & 3 \\
\hline & 5000 & & 100 & 100 & 100 & 6 & 6 & 6 & 4 & 4 & 3 \\
\hline
\end{tabular}

Table 2. Empirical sizes $(\times 100)$ of Mitchell separability test for $\beta=2$

For Shao and Li test the set $\Lambda$, vector $G$ and matrix $A$ are chosen for the 
combinations of lags $h_{1}=0, h_{2}=0,1$ and $u=1,2$ as follows

$$
\begin{aligned}
\Lambda & =\{(0,0,0),(0,1,1),(0,1,2),(0,1,0),(0,0,1),(0,0,2)\}, \\
G & =\{C(0,0,0), C(0,1,1), C(0,1,2), C(0,1,0), C(0,0,1), C(0,0,2)\}, \\
f(G) & =\left\{\frac{C(0,1,1)}{C(0,1,0)}, \frac{C(0,1,2)}{C(0,1,0)}, \frac{C(0,0,1)}{C(0,0,0)}, \frac{C(0,0,2)}{C(0,0,0)}\right\}
\end{aligned}
$$

Table 3. Empirical powers $(\times 100)$ of Mitchell separability test

\begin{tabular}{cccccccccc}
\hline \hline & \multicolumn{10}{c}{$T$} \\
\cline { 2 - 10 }$n$ & 5 & 10 & 30 & 40 & 50 & 60 & 100 & 300 & 500 \\
\hline 20 & 16 & - & - & - & - & - & - & - & - \\
50 & 45 & 45 & - & - & - & - & - & - & - \\
91 & 92 & 92 & 51 & - & - & - & - & - & - \\
100 & 94 & 58 & 78 & - & - & - & - & - & - \\
121 & 98 & 99 & 97 & 72 & - & - & - & - & - \\
151 & 100 & 100 & 100 & 99 & 93 & - & - & - & - \\
181 & 100 & 100 & 100 & 100 & 100 & 96 & - & - & - \\
300 & 100 & 100 & 100 & 100 & 100 & 100 & - & - & - \\
301 & 100 & 100 & 100 & 100 & 100 & 100 & 100 & - & - \\
500 & 100 & 100 & 100 & 100 & 100 & 100 & 100 & - & - \\
901 & 100 & 100 & 100 & 100 & 100 & 100 & 100 & 100 & - \\
1000 & 100 & 100 & 100 & 100 & 100 & 100 & 100 & 100 & - \\
1501 & 100 & 100 & 100 & 100 & 100 & 100 & 100 & 100 & 100 \\
2000 & 100 & 100 & 100 & 100 & 100 & 100 & 100 & 100 & 100 \\
\hline
\end{tabular}

and

$$
A_{1}=\left(\begin{array}{cccc}
1 & 0 & -1 & 0 \\
0 & 1 & 0 & -1
\end{array}\right)
$$

For degree of freedom $q=2$, and significance level of $\alpha=0.05$ the critical value from Lobato (2001) is equal to $U_{2,0.05}=103.6$. In a simulation study using the covariance functions (20) and (21) for different values of $a, b, \beta$ and $T$ the empirical sizes and powers of the tests are estimated for 100 iteration. Table 4 shows that when $T$ increases, the empirical sizes for $T S_{1}, T S_{2}$ and $\hat{\phi}$ increase and for different $a, b, \beta$ and $T$ the test statistics $T S_{1}$ and $T S_{2}$ have smaller empirical sizes than $\hat{\phi}$. For example for $a=2, b=2, \beta=0.1$ and $T=$ 20 we obtain $\hat{\phi}=0.68$ and $T S_{1}=T S_{2}=0$. However, for large $T$, the Shao and Li test has large empirical sizes but it has smaller empirical sizes than Fuentes' test. Table 5 shows that the empirical power of three test statistics 
are increased when the value of $T$ increases, but the test statistics $\hat{\phi}$ has larger power than $T S_{1}$ and $T S_{2}$ for all values of $T$. However the Fuentes' test has larger power, but dose not have valuable sizes. Thus, the test statistic of Shao and $\mathrm{Li}$ is preferred. Although there is no discordination between empirical sizes of $T S_{1}$ and $T S_{2}, T S_{1}$ has a larger power than $T S_{2}$. Regardingly; we can suggest that the $T S_{1}$ statistic is more suitable for assessment of separability of spatio-temporal data.

Table 4. Empirical sizes $(\times 100)$ of separability tests

\begin{tabular}{|c|c|c|c|c|c|c|c|c|c|c|c|c|c|c|}
\hline & & & \multicolumn{12}{|c|}{$T$} \\
\hline \multicolumn{3}{|c|}{ Parameter } & \multicolumn{3}{|c|}{20} & \multicolumn{3}{|c|}{50} & \multicolumn{3}{|c|}{100} & \multicolumn{3}{|c|}{300} \\
\hline$\beta$ & $a$ & $b$ & $T S_{1}$ & $T S_{2}$ & $\hat{\phi}$ & $T S_{1}$ & $T S_{2}$ & $\hat{\phi}$ & $T S_{1}$ & $T S_{2}$ & $\hat{\phi}$ & $T S_{1}$ & $T S_{2}$ & $\hat{\phi}$ \\
\hline \multirow[t]{9}{*}{0.1} & 0.1 & 0.1 & 1 & 0 & 21 & 0 & 0 & 29 & 6 & 3 & 29 & 7 & 9 & 23 \\
\hline & & 1 & 2 & 1 & 27 & 1 & 1 & 26 & 2 & 3 & 22 & 0 & 1 & 29 \\
\hline & & 2 & 0 & 1 & 40 & 0 & 0 & 31 & 0 & 0 & 33 & 2 & 2 & 20 \\
\hline & 1 & 0.1 & 2 & 5 & 33 & 11 & 14 & 42 & 22 & 20 & 39 & 36 & 30 & 38 \\
\hline & & 1 & 0 & 0 & 26 & 6 & 4 & 37 & 4 & 4 & 40 & 21 & 17 & 42 \\
\hline & & 2 & 1 & 1 & 55 & 1 & 1 & 62 & 3 & 3 & 52 & 20 & 16 & 42 \\
\hline & 2 & 0.1 & 3 & 4 & 43 & 18 & 15 & 61 & 38 & 31 & 77 & 65 & 53 & 91 \\
\hline & & 1 & 4 & 2 & 26 & 7 & 5 & 51 & 9 & 8 & 64 & 40 & 29 & 85 \\
\hline & & 2 & 0 & 0 & 68 & 2 & 3 & 80 & 12 & 12 & 86 & 38 & 30 & 84 \\
\hline \multirow[t]{9}{*}{1} & 0.1 & 0.1 & 2 & 3 & 17 & 2 & 2 & 26 & 4 & 5 & 20 & 15 & 15 & 31 \\
\hline & & 1 & 3 & 3 & 16 & 1 & 1 & 26 & 4 & 3 & 16 & 7 & 8 & 26 \\
\hline & & 2 & 2 & 4 & 18 & 1 & 1 & 13 & 4 & 4 & 12 & 4 & 4 & 19 \\
\hline & 1 & 0.1 & 8 & 10 & 24 & 20 & 20 & 33 & 32 & 23 & 41 & 63 & 62 & 83 \\
\hline & & 1 & 4 & 5 & 14 & 5 & & 517 & 21 & 21 & 27 & 38 & 35 & 25 \\
\hline & & 2 & 2 & 4 & 6 & 3 & 1 & 7 & 2 & 4 & 8 & 26 & 24 & 23 \\
\hline & 2 & 0.1 & 2 & 5 & 31 & 26 & 18 & 63 & 34 & 29 & 84 & 55 & 50 & 98 \\
\hline & & 1 & 2 & 3 & 16 & 14 & 9 & 38 & 31 & 30 & 57 & 56 & 40 & 82 \\
\hline & & 2 & 1 & 1 & 12 & 5 & 7 & 20 & 12 & 7 & 26 & 40 & 36 & 58 \\
\hline \multirow[t]{9}{*}{2} & 2 & 0.1 & 0 & 1 & 20 & 3 & 4 & 19 & 10 & 9 & 26 & 14 & 14 & 31 \\
\hline & & 1 & 0 & 3 & 15 & 0 & 0 & 25 & 5 & 6 & 25 & 13 & 13 & 25 \\
\hline & & 2 & 5 & 3 & 11 & 2 & 2 & 15 & 1 & 1 & 17 & 9 & 11 & 18 \\
\hline & 1 & 0.1 & 6 & 8 & 25 & 24 & 20 & 41 & 40 & 36 & 49 & 67 & 67 & 51 \\
\hline & & 1 & 3 & 3 & 19 & 12 & 10 & 26 & 26 & 18 & 34 & 45 & 44 & 35 \\
\hline & & 2 & 4 & 3 & 6 & 6 & 6 & 15 & 12 & 14 & 15 & 33 & 30 & 27 \\
\hline & 2 & 0.1 & 5 & 7 & 37 & 17 & 11 & 70 & 35 & 28 & 82 & 56 & 64 & 95 \\
\hline & & 1 & 2 & 1 & 27 & 20 & 15 & 61 & 39 & 33 & 68 & 59 & 49 & 91 \\
\hline & & 2 & 1 & 0 & 7 & 8 & 6 & 18 & 24 & 18 & 49 & 51 & 38 & 84 \\
\hline
\end{tabular}


Table 5. Empirical powers $(\times 100)$ of separability tests

\begin{tabular}{cccc}
\hline \hline$T$ & $\hat{\phi}$ & $T S_{1}$ & $T S_{2}$ \\
\hline 20 & 45 & 21 & 15 \\
50 & 94 & 55 & 40 \\
100 & 100 & 85 & 77 \\
300 & 100 & 98 & 98 \\
500 & 100 & 100 & 99 \\
\hline
\end{tabular}

\section{Evaluation of Axial Symmetry Tests}

In this section, the spectral test of Park and Fuentes (2008) and nonparametric test of Shao and $\mathrm{Li}$ (2009) under axial symmetry in space and time are compared based on empirical sizes and powers. In this simulation study, the asymmetric exponential spatio-temporal covariance function of

$$
C(\mathbf{h}, u)=\sigma_{0} I_{\{(\mathbf{h})=u=0\}}(\mathbf{h}, u)+\sigma_{1} \exp \left\{-\sqrt{\beta^{2}(u-\mathbf{h} \nu)^{2}+\alpha^{2}\|\mathbf{h}\|^{2}},\right.
$$

is considered, where $\sigma_{0}=0.01, \sigma_{1}=1, \alpha$ and $\beta$ show the decaying rates of spatial and temporal correlations respectively. Here, the vector $\nu=\left(\nu_{1}, \nu_{2}\right) \in$ $R^{2}$ controls the asymmetry of the spatial-temporal covariance function. For example $\nu_{i}=0$ yields a covariance that is $i$-axial symmetry in space or as another example; for $\nu=(0,0)$ the covariance is symmetry in space and time.

To compare Park and Fuentes test with Shao and Li test for lack of (1) axial symmetry in space, four pairs of $\left\{\left(a_{2}^{i}, t^{\mathbf{a}_{i}}\right),\left(b_{2}^{i}, t^{\mathbf{b}_{i}}\right)\right\}$ are considered, where $a_{2}^{i}$ and $b_{2}^{i}$ are the second spatial coordinates on a $30 \times 30$ regular spatial grid and $t^{a_{2}^{i}}$ and $t^{b_{2}^{i}}$ are times. To obtain approximately uncorrelated estimates, the spatial pairs and frequencies are chosen in such a way that the spatial and temporal lags are at least 15 and $\frac{\pi}{5}$, respectively. It is obvious that the temporal lags are bigger than, $\frac{\pi}{6}$, the bandwidth of the function $W_{s}(\alpha)$ in $(22)$. Each frequency $\omega_{j}$ was chosen as $\omega_{j}=\frac{\pi(2(j-1)+1)}{10}$. The estimate $\hat{\phi}_{\mathbf{a b}}(\omega)$ was evaluated at the spatial frequencies, $\omega_{1}=\frac{\pi}{10}, \omega_{2}=\frac{3 \pi}{10}$, $\omega_{3}=\frac{5 \pi}{10}, \omega_{4}=\frac{7 \pi}{10}, \omega_{5}=\frac{9 \pi}{10}$ and spatial pairs as
$(20,118)$ 
The random field $Z$ is simulated for $N=120, T=200$ from $N_{120 \times 200}(0, \Sigma)$. Since $\Sigma$ is of dimension $120 T \times 120 T$ and $Z$ is of length $120 T$, we split the whole random field of $Z$ into $Z_{1}, Z_{2}, \ldots, Z_{[n / k]}$, which of them is of size $120 \times k$. In fact assume that only the nearest neighbors of $Z_{i}$ are temporally correlated. This assumption is justified by the negligible temporal correlation, when the time lag exceeds a certain value of $k$. An initial Gaussian random field $Z_{1}$ is generated, then for given $Z_{1}=z_{1}$ generate $Z_{2}\left|\left(Z_{1}=z_{1}\right), Z_{3}\right|\left(Z_{2}=z_{2}\right)$, and so on. Here for $k=20, h_{1}=1,2, h_{2}=1$ and $u=1$, we choose

$$
\begin{aligned}
& \Lambda=\{(1,1,1),(-1,1,1),(2,1,1),(-2,1,1)\}, \\
& G=(C(1,1,1), C(-1,1,1), C(2,1,1), C(-2,1,1)),
\end{aligned}
$$

and

$$
A_{1}=\left(\begin{array}{cccc}
1 & -1 & 0 & 0 \\
0 & 0 & 1 & -1
\end{array}\right)
$$

So the degrees of freedom and critical value are $q=2, U_{2,0.05}=103.6$, respectively and the test statistic simplifies as

$$
T S=\left|T_{n}\right|\left\{A \widetilde{G}_{N}\right\}^{\prime} \widetilde{V}_{N}^{-1}\left\{A \widetilde{G}_{N}\right\},
$$

where the covariance function under 1-axial symmetry in space is estimated by

$$
\widetilde{C}_{J}\left(-h_{1}, h_{2}, u\right)=\frac{\sum_{\mathbf{s} \in S(\mathbf{h})} \sum_{t=1}^{J} Z(\mathbf{s}, t) Z\left(s_{1}-h_{1}, s_{2}+h_{2}, t+u\right)}{|S(\mathbf{h})|\left|T_{n}\right|} .
$$

If $\nu_{1}=0$, the covariance function (24) is 1-axial symmetry in space given by

$$
C(\mathbf{h}, u)=\sigma_{0} I_{\{\mathbf{h}=u=0\}}(\mathbf{h}, u)+\sigma_{1} \exp \left\{-\sqrt{\beta^{2}\left(u-h_{2} \nu_{2}\right)^{2}+\alpha^{2}\|\mathbf{h}\|^{2}}\right\} .
$$

In a simulation study the covariance function (25) is used with different values of $\alpha, \beta, \nu_{2}$ and $T$ to estimate the empirical size. The covariance function (24) with $\nu_{2}=0.5$ and different values of $\alpha, \beta, \nu_{1}$ and $T$ are used to estimate the empirical power for 100 iterations. The nominal level is set to be 0.05. Results in Tables 6 and 7 show that changes of $\alpha, \beta$ and $\nu_{2}$ do not affect on empirical sizes. But test of Shao and Li has smaller empirical sizes. The empirical power decreases as $\alpha$ increases and it increases as $\nu_{1}$ and $\beta$ increase. Thus the test has smaller power for $\nu_{1}=0.05$. Since for 
$\alpha=0.02$ and 1 the Shao and Li test has larger power and for $\alpha=2$ there is no difference between power of two tests and according to the empirical sizes, the Shao and Li test can be preferred for testing the spatial axial symmetry.

Table 6. Empirical sizes $(\times 100)$ of 1-axial symmetry in space test

\begin{tabular}{|c|c|c|c|c|}
\hline \multicolumn{3}{|c|}{ Parameter } & \multirow[b]{2}{*}{$\phi$} & \multirow[b]{2}{*}{$T S$} \\
\hline$\alpha$ & $\beta$ & $\nu_{2}$ & & \\
\hline \multirow{6}{*}{0.02} & 0.75 & 0.05 & 7 & 1 \\
\hline & & 0.5 & 3 & 8 \\
\hline & 1 & 0.05 & 6 & 2 \\
\hline & & 0.5 & 9 & 3 \\
\hline & 2 & 0.05 & 9 & 4 \\
\hline & & 0.5 & 8 & 4 \\
\hline \multirow[t]{6}{*}{1} & 0.75 & 0.05 & 6 & \\
\hline & & 0.5 & 9 & 5 \\
\hline & 1 & 0.05 & 5 & 5 \\
\hline & & 0.5 & 7 & 6 \\
\hline & 2 & 0.05 & 3 & 8 \\
\hline & & 0.5 & 5 & 7 \\
\hline \multirow[t]{6}{*}{2} & 0.75 & 0.05 & 8 & 7 \\
\hline & & 0.5 & 7 & 4 \\
\hline & 1 & 0.05 & 6 & 6 \\
\hline & & 0.5 & 6 & 5 \\
\hline & 2 & 0.05 & 2 & 6 \\
\hline & & 0.5 & 6 & 3 \\
\hline
\end{tabular}


Table 7. Empirical powers $(\times 100)$ of 1 -axial symmetry in space test

\begin{tabular}{|c|c|c|c|c|}
\hline \multicolumn{3}{|c|}{ Parameter } & \multirow[b]{2}{*}{$\phi$} & \multirow[b]{2}{*}{$T S$} \\
\hline$\alpha$ & $\beta$ & $\nu_{1}$ & & \\
\hline \multirow[t]{6}{*}{0.02} & 0.75 & 0.05 & 15 & 100 \\
\hline & & 0.5 & 85 & 100 \\
\hline & 1 & 0.05 & 10 & 100 \\
\hline & & 0.5 & 90 & 100 \\
\hline & 2 & 0.05 & 5 & 100 \\
\hline & & 0.5 & 95 & 99 \\
\hline \multirow[t]{6}{*}{1} & 0.75 & 0.05 & 4 & 11 \\
\hline & & 0.5 & 4 & 40 \\
\hline & 1 & 0.05 & 11 & 10 \\
\hline & & 0.5 & 3 & 66 \\
\hline & 2 & 0.05 & 4 & 10 \\
\hline & & 0.5 & 4 & 100 \\
\hline \multirow[t]{6}{*}{2} & 0.75 & 0.05 & 4 & 4 \\
\hline & & 0.5 & 4 & 6 \\
\hline & 1 & 0.05 & 6 & 9 \\
\hline & & 0.5 & 2 & 7 \\
\hline & 2 & 0.05 & 1 & 6 \\
\hline & & 0.5 & 3 & 19 \\
\hline
\end{tabular}

To compare the Park and Fuentes test with Shao and Li test for lack of axial symmetry in time the following pairs on a $60 \times 60$ regular spatial grid are considered.

$$
\begin{array}{ll}
\mathbf{a}_{1}=(3,4) & \mathbf{b}_{1}=(5,4) \\
\mathbf{a}_{2}=(21,4) & \mathbf{b}_{2}=(23,4) \\
\mathbf{a}_{3}=(39,4) & \mathbf{b}_{3}=(41,4) \\
\mathbf{a}_{4}=(3,22) & \mathbf{b}_{4}=(5,22) \\
\mathbf{a}_{5}=(21,22) & \mathbf{b}_{5}=(23,22) \\
\mathbf{a}_{6}=(39,22) & \mathbf{b}_{6}=(41,22) .
\end{array}
$$

For $h_{1}=2,16, h_{2}=18$ and $u=1,2$ we have

$$
\begin{aligned}
\Lambda= & (2,18,1),(2,18,-1),(16,18,1),(16,18,-1),(2,18,2),(2,18,-2), \\
& (16,18,2),(16,18,-2)\}, \\
G= & (C(2,18,1), C(2,18,-1), C(16,18,1), C(16,18,-1), C(2,18,2), \\
& C(2,18,-2), C(16,18,2), C(16,18,-2))
\end{aligned}
$$


and

$$
A_{1}=\left(\begin{array}{ccccccc}
1 & -1 & 0 & 0 & 0 & 0 & 0 \\
0 & 0 & 1 & -1 & 0 & 0 & 0 \\
0 & 0 & 0 & 0 & 1 & -1 & 0 \\
0 & 0 & 0 & 0 & 0 & 1 & -1
\end{array}\right)
$$

Therefore $q=4, U_{4,0.05}=259.3$ and the test statistic simplify as

$$
T S=\left|T_{n}\right|\left\{A \widetilde{G}_{N}\right\}^{\prime} \widetilde{V}_{N}^{-1}\left\{A \widetilde{G}_{N}\right\},
$$

where the covariance function under axial symmetry in time is estimated by

$$
\widetilde{C}_{J}(\mathbf{h},-u)=\frac{\sum_{\mathbf{s} \in S(\mathbf{h})} \sum_{t=480-j+1}^{480} Z(\mathbf{s}, t) Z(\mathbf{s}+\mathbf{h}, t-u)}{|S(\mathbf{h})|\left|T_{n}\right|} .
$$

If $\nu=0$, the covariance function (24) is axial symmetry in time is given by

$$
C(\mathbf{h}, u)=\sigma_{0} I_{\{\mathbf{h}=u=0\}}(\mathbf{h}, u)+\sigma_{1} \exp \left\{-\sqrt{\beta^{2} u^{2}+\alpha^{2}\|\mathbf{h}\|^{2}}\right\} .
$$

In a simulation study the covariance function (26) is used with different values of $\alpha, \beta$ and $T$ to estimate the empirical size. The covariance function (24) with $\nu_{2}=0.5$ and different values of $\alpha, \beta, \nu_{1}$ and $T$ are used to estimate the empirical power for 100 iterations. The nominal level is set to be 0.05 . Results in Tables 8 and 9 show that changes of $\alpha$ and $\beta$ do not affect on empirical sizes. For $\alpha=0.02$ the Shao and Li test has a smaller empirical sizes, than the others. For $\alpha=1$ and 2 both tests are roughly equivalent. For $\alpha=0.02$ the Fuentes test has larger empirical power, but for $\alpha=1$ and 2 there is no meaningful difference between two tests. According to empirical sizes and powers, the Fuentes test can be preferred for testing the axial symmetry in time.

\section{Numerical Example}

The wind-speed data used by Cressie and Huang (1999) for mapping the eastwest component of the wind speed, which has been measured over a region in the tropical western Pasific Ocean is explored in this example. These data are given on a regular spatio-temporal grid of $17 \times 17$ sites with grid spacing of about $210 \mathrm{~km}$ for every 6 hours starting from November 1992 through February 1993. So there are 289 space locations and also 480 time locations, 
where a positive value represents speed of east wind and a negative value represents speed of west wind. Cressie and Huang (1999), in an exploratory data analysis showed that these data are strongly spatio-temporal correlated and homoscedastic. Time series plots for two locations of $(13,5),(13,13)$ in Figure 1 indicate existence of temporal trend. So the first step has been taken by detrending the data.

The Shao and Li nonparametric test has been used for separability of the covariance function based on six combination of space lags $\|\mathbf{h}\|=0,1$ and time lags $u=1,2, \Lambda, G$ and $f(G)$ have been chosen to be as followed;

$$
\begin{aligned}
\Lambda & =\{(0,0,0),(0,1,1),(0,1,2),(0,1,0),(0,0,1),(0,0,2)\} \\
G & =(C(0,0,0), C(0,1,1), C(0,1,2), C(0,1,0), C(0,0,1), C(0,0,2)) \\
f(G) & =\left(\frac{C(0,1,1)}{C(0,1,0)}, \frac{C(0,1,2)}{C(0,1,0)}, \frac{C(0,1,0)}{C(0,0,0)}, \frac{C(0,0,2)}{C(0,0,0)}\right) .
\end{aligned}
$$

\begin{tabular}{|c|c|c|c|c|c|c|c|c|c|c|}
\hline \multirow{2}{*}{\multicolumn{2}{|c|}{ Parameter }} & \multicolumn{9}{|c|}{$T$} \\
\hline & & \multicolumn{2}{|c|}{20} & \multirow[b]{2}{*}{$\hat{\phi} T S$} & \multicolumn{2}{|c|}{30} & \multicolumn{2}{|c|}{50} & \multicolumn{2}{|c|}{100} \\
\hline$\alpha$ & $\beta$ & $\hat{\phi} T S$ & & & & $\hat{\phi} T S$ & $\hat{\phi} T S$ & & & \\
\hline \multirow[t]{3}{*}{0.02} & 0.75 & 11 & 6 & & 9 & 2 & 6 & 3 & 11 & 2 \\
\hline & 1 & 9 & 7 & & 14 & 0 & 5 & 5 & 11 & 5 \\
\hline & 2 & 6 & 7 & & 11 & 7 & 7 & 3 & 6 & 6 \\
\hline \multirow[t]{3}{*}{1} & 0.75 & 5 & 7 & & 5 & 9 & 6 & 2 & 9 & 5 \\
\hline & 1 & 5 & 4 & & 9 & 6 & 2 & 5 & 5 & 6 \\
\hline & 2 & 8 & 9 & & 12 & 6 & 7 & 8 & 1 & ? \\
\hline \multirow[t]{3}{*}{ ] 2} & 0.75 & 5 & 6 & & 2 & 6 & 7 & 4 & 4 & 1 \\
\hline & 1 & 1 & 10 & & 7 & 3 & 2 & 4 & 11 & \\
\hline & 2 & 3 & 4 & & 8 & 9 & 7 & 1 & 4 & \\
\hline
\end{tabular}

Table 8 . Empirical sizes $(\times 100)$ of 1-axial symmetry in space test 
Table 9. Empirical powers $(\times 100)$ of 1 -axial symmetry in space test

\begin{tabular}{|c|c|c|c|c|c|c|c|c|c|c|c|}
\hline & & & \multicolumn{9}{|c|}{$T$} \\
\hline \multicolumn{3}{|c|}{ Parameter } & \multicolumn{2}{|c|}{20} & \multirow[b]{2}{*}{$\hat{\phi} T S$} & \multicolumn{2}{|c|}{30} & \multicolumn{2}{|c|}{50} & \multicolumn{2}{|c|}{100} \\
\hline$\alpha$ & $\beta$ & $\nu_{1}$ & $\hat{\phi} T S$ & & & & $\hat{\phi} T S$ & $\hat{\phi} T S$ & & & \\
\hline \multirow[t]{6}{*}{0.02} & 0.75 & 0.05 & 14 & 10 & & 20 & 4 & 15 & 5 & 33 & 3 \\
\hline & & 0.5 & 96 & 10 & & 100 & 11 & 100 & 15 & 100 & 6 \\
\hline & 1 & 0.05 & 9 & 8 & & 16 & 5 & 28 & 1 & 44 & 6 \\
\hline & & 0.5 & 94 & 12 & & 95 & 13 & 100 & 8 & 100 & 14 \\
\hline & 2 & 0.05 & 11 & 9 & & 14 & 9 & 16 & 5 & 21 & 6 \\
\hline & & 0.5 & 97 & 14 & & 98 & 9 & 100 & 14 & 100 & 15 \\
\hline \multirow[t]{6}{*}{1} & 0.75 & 0.05 & 5 & 5 & & 4 & 5 & 4 & 6 & 5 & 7 \\
\hline & & 0.5 & 8 & 7 & & 8 & 5 & 6 & 2 & 13 & 3 \\
\hline & 1 & 0.05 & 5 & 10 & & 9 & 6 & 7 & 5 & 6 & 4 \\
\hline & & 0.5 & 9 & 6 & & 5 & 7 & 6 & 3 & 5 & 2 \\
\hline & 2 & 0.05 & 6 & 5 & & 4 & 2 & 5 & 4 & 3 & 7 \\
\hline & & 0.5 & 3 & 6 & & 6 & 7 & 5 & 9 & 10 & 7 \\
\hline \multirow[t]{6}{*}{2} & 0.75 & 0.05 & 6 & 4 & & 4 & 6 & 4 & 2 & 5 & 3 \\
\hline & & 0.5 & 5 & 6 & & 3 & 3 & 7 & 6 & 5 & 6 \\
\hline & 1 & 0.05 & 8 & 7 & & 5 & 3 & 3 & 4 & 3 & 9 \\
\hline & & 0.5 & 11 & 11 & & 10 & 7 & 5 & 6 & 5 & 2 \\
\hline & 2 & 0.05 & 7 & 6 & & 1 & 7 & 3 & 3 & 9 & 2 \\
\hline & & 0.5 & 4 & 12 & & 5 & 11 & 8 & 4 & 5 & 4 \\
\hline
\end{tabular}

Since the test statistic value of 241.21 exceeds the critical value $U_{2,0.05}=$ 103.6 (tabled in Lobato, 2001), it is concluded that the spatio-temporal covariance function of the data is not separable in nominal level 0.05. In order to assess the axial symmetry in space by Shao and Li (2009) nonparametric test; based on eight combination of space lags, $\|\mathbf{h}\|=\sqrt{2}, \sqrt{5}, \sqrt{8}$ and time lag $\mathrm{u}=1, \Lambda$ and $G$ have been chosen to be as followed;

$$
\begin{aligned}
\Lambda= & \{(1,2,1),(-1,-2,1),(1,1,1),(-1,-1,1),(2,2,1),(-2,-2,2), \\
& (2,1,1),(-2,-1,1)\} \\
G= & (C(1,2,1), C(-1,-2,1), C(1,1,1), C(-1,-1,1), C(2,2,1), \\
& C(-2,-2,2), C(2,1,1), C(-2,-1,1)) .
\end{aligned}
$$

Since the test statistic value of 24.35 is smaller than $U_{4,0.05}=259.3$, the spatio-temporal covariance function of wind-speed data has an axial symmetry in space. For testing axial symmetry in time using the Park and Fuentes (2008) test, $B_{T}=\frac{1}{10}$ is choosen. To obtain approximately uncorrelated estimates the time frequencies was considered to be (23) with distance $\frac{3 \pi}{4}$, which 
is larger than $\pi 5$, and four spatial pairs as

$$
\begin{array}{ll}
\mathbf{a}_{1}=(1,1) & \mathbf{b}_{1}=(3,1) \\
\mathbf{a}_{2}=(15,1) & \mathbf{b}_{2}=(17,1) \\
\mathbf{a}_{3}=(1,17) & \mathbf{b}_{3}=(3,17) \\
\mathbf{a}_{4}=(15,17) & \mathbf{b}_{4}=(17,17) .
\end{array}
$$
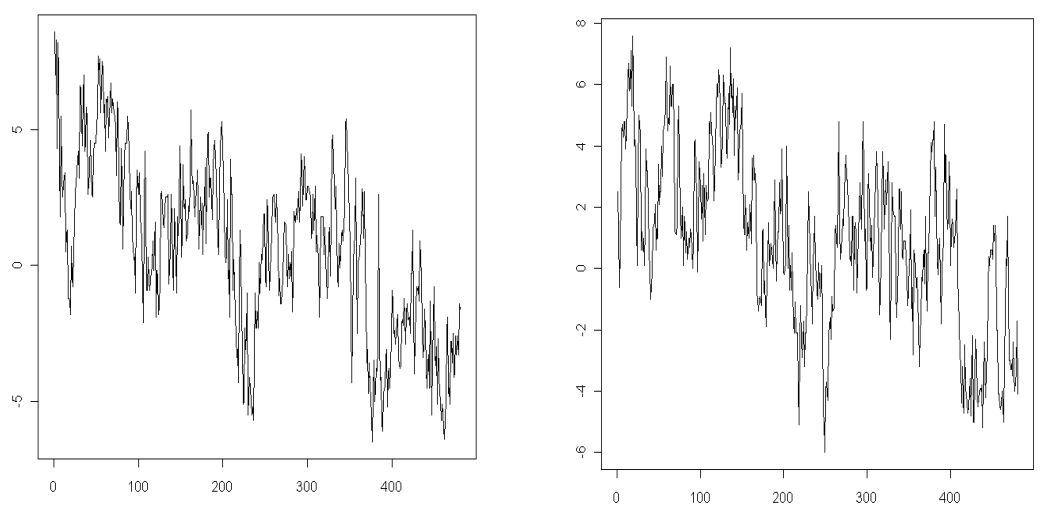

Figure 1. Time series plot of wind speed for sites $(13,5)$ and $(13,13)$

Since the obtained p-value for the null hypothesis $\beta_{j}=0$ is .034, the null hypothesis is significant. Therefore the spatio-temporal covariance function of the wind-speed data is not temporally axial symmetry in nominal level 0.05. Consequently a spatio-temporal covariance function which is nonseparable, axial asymmetric in time and axial symmetric in space would be needed to determine the correlation structure of the wind-speed data.

\section{Discussion}

In this article different methodology for testing symmetry and separability of spatio-temporal covariance functions are presented. Although the Mitchel likelihood ratio test for separability has large power for respectively large $T$ it does not have an empirical size and can not add trust to the results; this test is preferred for small $T$. Comparison of Shao and Li's nonparametric test with Fuentes' spectral test for separability of covariance function showed empirical sizes of tests have different meanings for some values of $T$, so for this reason Shao and Li's nonparametric test can be preferred for testing the 
separability. For axial symmetry in space and time tests, Fuentes' spectral test and Shao and Li nonparametric test have valuable empirical sizes and there is no difference between two tests, but according to empirical powers; Fuentes' spectral test for testing the axial symmetry in time and Shao and Li's nonparametric test for testing the axial symmetry in space can be preferred. When the separability hypothesis for a spatio-temporal data set is rejected, a nonseparable covariance function should be used for modeling the correlation structure of the data. Cressie and Huang (1999) and Gneiting (2002) proposed some nonseparable covariance models for spatio-temporal data. But Kent et al. (2011) showed that in certain circumstances most of these models possess a counterintuitive "dimple", which detracts from their modeling appeal, so the implication requires more attention.

\section{Acknowledgement}

The authors would like to thank the referees for their valuable comments and suggestions. Also partial support from Ordered and Spatial Data Center of Excellence of Ferdowsi University of Mashhad is acknowledged.

\section{References}

Cressie, N. and Huang, H.C. (1999). Classes of Nonseparable, Spatio-Temporal Stationary Covariance Functions, Journal of the American Statistical Association, 94, 1330-1340.

Fuentes, M. (2006). Testing for Separability of Spatial-Temporal Covariance Functions, Journal of Statistical Planning and Inference, 136, 447-466.

Gneiting, T. (2002). Nonseparable, Stationary Covariance Functions for Space-time Data, Journal of American Statistical Association 97, 590-600.

Haslett, J. and Raftery, A.E. (1989). Space-Time Modelling with Long-Memory Dependence: Assessing Irelands Wind-Power Resource (with discussion), Applied Statistics, 38, 1-50.

Kent, J.T., Mohammadzadeh, M. and Mohammadian, M.A. (2011). The Dimple in Gneiting's Spatial-Temporal Covariance Model, Biometrika, 98, 489-494.

Li, B., Genton, M.G. and Sherman, M. (2007). A Nonparametric Assessment of Properties of Space-Time Covariance Function, Journal of American Statistical Association, 102, 736-744.

Lobato, I.N. (2001). Testing that a Dependent Process is Uncorrelated, Journal of the American Statistical Association, 96, 1066-1076. 
Lu, N. and Zimmermann, D.L. (2005). The Likelihood Ratio Test for a Separable Covariance Matrix, Statistics and Probability Letters, 73, 449-457.

Mitchell, M.W., Genton, M.G. and Gumpertz, M.L. (2006), A likelihood Ratio Test for Separability of Covariances, Journal of Multivariate Analysis, 97, 1025-1043.

Shao, X. and Li, B. (2009). A Tuning Parameter Free Test for Properties of Space-Time Covariance Functions, Journal of Statistical Planing and Inference, 139, 4031-4038.

\section{Elham Behshad}

Department of Statistics, Tarbiat Modares University, Tehran, Iran.

\author{
Mohsen Mohammadzadeh \\ Department of Statistics, \\ Tarbiat Modares University, \\ Tehran, Iran. \\ email:mohsen_m@modares.ac.ir
}

